\title{
Space-Time Correlation Properties of a 3D Two-Sphere Model for Non-Isotropic MIMO Mobile-to-Mobile Channels
}

\author{
Yi Yuan ${ }^{1}$, Xiang Cheng ${ }^{2}$, Cheng-Xiang Wang ${ }^{1}$, David I. Laurenson ${ }^{3}$, Xiaohu $\mathrm{Ge}^{4}$, and Feng Zhao ${ }^{5}$ \\ ${ }^{1}$ Joint Research Institute for Signal and Image Processing, Heriot-Watt University, Edinburgh EH14 4AS, UK. \\ ${ }^{2}$ School of Electronics Engineering and Computer Science, Peking University, Beijing 100871, P. R. China. \\ ${ }^{3}$ Joint Research Institute for Signal and Image Processing, University of Edinburgh, Edinburgh EH9 3JL, UK. \\ ${ }^{4}$ Department of Electronics and Information Engineering, Huazhong University of Science and Technology, Wuhan 430074, P. R. China. \\ ${ }^{5}$ Department of Science and Technology, Guilin University of Electronic Technology, Guilin 541004, P. R. China. \\ Email: yy120@hw.ac.uk, xiangcheng.86@googlemail.com, cheng-xiang.wang@hw.ac.uk, dave.laurenson@ed.ac.uk, \\ xhge@mail.hust.edu.cn, zhaofeng@guet.edu.cn
}

\begin{abstract}
This paper proposes a novel three-dimensional (3D) two-sphere regular-shaped geometry-based stochastic model (RSGBSM) with only double-bounced rays for non-isotropic scattering narrowband multiple-input multiple-output (MIMO) mobileto-mobile (M2M) channels. The proposed 3D model has the ability to investigate the joint impact of both the azimuth angle and elevation angle on channel statistics. Based on the proposed model, the space-time (ST) correlation function (CF) is derived and the impact of some important parameters on the resulting ST CF is investigated. Numerical results show that the 3D model results in lower ST correlations than the corresponding $2 \mathrm{D}$ model.
\end{abstract}

\section{INTRODUCTION}

In recent years, M2M communications have been encountered in many new applications, such as wireless mobile ad hoc peer-to-peer networks, cooperative systems [1], and intelligent transportation systems. In a M2M communication system, both the transmitter $(\mathrm{Tx})$ and the receiver $(\mathrm{Rx})$ are in motion often with low elevation antennas. This is different from conventional fixed-to-mobile (F2M) cellular radio systems [2], where only one terminal moves. Therefore, existing channel models for F2M communications cannot be used directly for the design of M2M communication systems. M2M channel modeling has become a hot research topic.

RS-GBSMs have been widely used to mimic F2M [2] and M2M channels [3]. Akki and Haber were the first to propose a RS-GBSM for isotropic scattering single-input single-output (SISO) M2M Rayleigh fading channels in [4] and [5]. In [6], a two-ring RS-GBSM considering only double-bounced rays was presented for non-isotropic scattering MIMO M2M Rayleigh fading channels. In [7], Zajic̀ and Stüber proposed a more general two-ring RS-GBSM that constructs the received complex fading envelope as a superposition of the line-ofsight (LoS), single-bounced, and double-bounced rays. More recently, in order to investigate the impact of vehicular traffic density on channel statistics, new RS-GBSMs for nonisotropic scattering MIMO M2M channels were proposed in [8].

However, the above reported models assumed that the fields incident on the Tx or Rx antennas are composed of a number of waves traveling only in the horizontal plane. Therefore, only the impact of azimuth angle on channel statistics was considered in those models. This assumption is valid only for certain environments, e.g., rural scenarios. While for urban environments, the impact of elevation angle on channel statistics has to be considered. In [9], the authors proposed a 3D two-cylinder RS-GBSM for non-isotropic scattering narrowband MIMO M2M channels. This narrowband 3D model was further extended into a wideband one in [10]. However, both the aforementioned narrowband and wideband 3D RS-GBSMs assumed the azimuth angle and elevation angle are completely independent and thus analyze them separately. This definitely reduces the reality (accuracy) of the 3D models in [9] and [10].

To remedy the above mentioned common drawback of available 3D RS-GBSMs, in this paper we propose a novel 3D two-sphere non-isotropic scattering narrowband MIMO M2M RS-GBSM that assumes all effective scatterers are located on two spheres, one around the Tx and the other around the Rx. The proposed model is a simplified model and focuses on ST correlation properties compared to the model proposed in [11]. By using the Von Mises Fisher (VMF) distribution [12] to characterize the distribution of the effective scatterers, the proposed 3D model can investigate channel statistics with the consideration of the relationship between the azimuth angle and the elevation angle. This means the proposed 3D RS-GBSM has the ability to jointly study the impact of the azimuth angle and elevation angle on channel statistics. Furthermore, based on the proposed 3D model, we derive the ST CF for non-isotropic scattering MIMO M2M channels. Finally, from the derived $\mathrm{CF}$, we investigate and analyze the impact of some important parameters, e.g., environment parameters and elevation angles, on correlation properties of non-isotropic scattering 3D MIMO M2M channels.

The remainder of this paper is structured as follows. Section II describes the novel 3D two-sphere RS-GBSM for non-isotropic scattering narrowband MIMO M2M channels. In Section III, the ST correlation functions are derived. The corresponding numerical results and analysis are presented in Section IV. Finally, conclusions are educed in Section V.

\section{A Novel 3D Two-Sphere MIMO M2M RS-GBSM}

Let us consider a narrowband MIMO M2M communication system with $L_{T}$ transmit and $L_{R}$ receive omnidirectional 
antenna elements. Both the Tx and $\mathrm{Rx}$ are in motion and equipped with low elevation antennas. The radio propagation environment is characterized by $3 \mathrm{D}$ scattering with non lineof-sight (NLoS) propagation conditions between the Tx and Rx. The MIMO channel is described by an $L_{R} \times L_{T}$ matrix $\mathbf{H}(t)=\left[h_{i j}(t)\right]_{L_{R} \times L_{R}}$ of complex fading envelopes.

Fig. 1. shows the proposed novel 3D two-sphere RS-GBSM for MIMO M2M channels, with $L_{R}=L_{T}=2$ antenna elements as an example. The two-sphere model defines two spheres, one around the Tx with radius $R_{T}$ and the other around the $\mathrm{Rx}$ with radius $R_{R}$. Around the transmitter, $M$ fixed omnidirectional scatterers lie on the surface of a sphere and the $m^{t h}$ transmit scatterer is defined by $S_{T}^{(m)}$. Similarly, around the receiver, $N$ fixed omnidirectional scatterers lie on the surface of a sphere and the $n^{\text {th }}$ receive scatterer is denoted by $S_{R}^{(n)}$. The parameters in Fig. 1 are defined in Table 1 .

It is normally assumed that the radius $R_{T}$ and $R_{R}$ are much smaller than the distance $D$, i.e., $\max \left\{R_{T}, R_{R}\right\}<<D$ (local scattering condition). Furthermore, in order to avoid the channel experiencing keyhole behavior [9], we assume that the distance $D$ is smaller than $\frac{4 R_{T} R_{R} L_{R}}{\lambda\left(L_{T}-1\right)\left(L_{R}-1\right)}$, where $\lambda$ denotes the carrier wavelength. Finally, it is reasonable to assume that the antenna element spacing $d_{T}(p, \tilde{p})$ at the Tx and $d_{R}(q, \tilde{q})$ at the $\mathrm{Rx}$ are much smaller than the radius $R_{T}$ and $R_{R}$, i.e., $\max \left\{d_{T}(p, \tilde{p}), d_{R}(q, \tilde{q})\right\}<<\min \left\{R_{T}, R_{R}\right\}$.

In the 3D two-sphere model, the number of local scatterers around the Tx and Rx is assumed to be infinite. The received complex fading envelope of the link $A_{T}^{(p)}-A_{R}^{(q)}$ is

$$
\begin{aligned}
h_{p q}(t)= & \lim _{M, N \rightarrow \infty} \frac{1}{\sqrt{M N}} \sum_{m=1}^{M} \sum_{n=1}^{N}\left[e^{-j \frac{2 \pi}{\lambda}\left(\varepsilon_{p m}+\varepsilon_{m n}+\varepsilon_{n q}\right)}\right. \\
& \times e^{j \phi_{m n}+j 2 \pi t f_{T \max } \cos \left(\alpha_{T}^{(m)}-\gamma_{T}\right) \cos \beta_{T}^{(m)}} \\
& \left.\times e^{j 2 \pi t f_{R \max } \cos \left(\alpha_{R}^{(n)}-\gamma_{R}\right) \cos \beta_{R}^{(n)}}\right]
\end{aligned}
$$

where $f_{T \max }=\frac{v_{T}}{\lambda}$ and $f_{R \max }=\frac{v_{R}}{\lambda}$ are the maximum Doppler frequencies associated with the Tx and Rx, respectively. Also, it is assumed that the phases $\phi_{m n}$ are random variables uniformly distributed in the interval $[-\pi, \pi)$ and independent from the angles of departure (AoDs) and angles of arrival (AoAs).

From Fig. 1, the distances $\varepsilon_{p m}, \varepsilon_{\tilde{p} m}, \varepsilon_{m n}, \varepsilon_{\tilde{q} n}, \varepsilon_{q n}$ can be expressed as functions of the random angles $\alpha_{T}^{(m)}, \alpha_{R}^{(n)}, \beta_{T}^{(m)}$, $\beta_{R}^{(n)}$ as follows:

$$
\begin{aligned}
\varepsilon_{m n} & \approx \sqrt{D^{2}+\left(h_{T}-h_{R}\right)^{2}} \approx D \\
\varepsilon_{p m} & \approx R_{T}-d_{T}\left[\cos \beta_{T} \cos \phi_{T} \cos \left(\theta_{T}-\alpha_{T}\right)\right. \\
& \left.+\sin \phi_{T} \sin \beta_{T}\right] \\
\varepsilon_{q n} & \approx R_{R}-d_{R}\left[\cos \beta_{R} \cos \phi_{R} \cos \left(\theta_{R}-\alpha_{R}\right)\right. \\
& \left.+\sin \phi_{R} \sin \beta_{R}\right] \\
\varepsilon_{\tilde{p} m} & \approx R_{T}+d_{T}\left[\cos \beta_{T} \cos \phi_{T} \cos \left(\theta_{T}-\alpha_{T}\right)\right. \\
& \left.+\sin \phi_{T} \sin \beta_{T}\right] \\
\varepsilon_{\tilde{q} n} & \approx R_{R}+d_{R}\left[\cos \beta_{R} \cos \phi_{R} \cos \left(\theta_{R}-\alpha_{R}\right)\right. \\
& \left.+\sin \phi_{R} \sin \beta_{R}\right] .
\end{aligned}
$$

The above distances are obtained based on the application of the law of cosines in appropriate triangles and small angle approximation (i.e., $\sin x \approx x$ and $\cos x \approx 1$ for small $x$ ), while the detailed derivations are omitted here for brevity.

\section{iII. Space-Time Correlation Properties of Mimo M2M CHANNELS}

\section{A. Space-Time Correlation Functions}

Based on the proposed 3D two-sphere RS-GBSM in (1), we will derive the ST CF for a non-isotropic scattering environment. The normalized ST CF between two complex fading envelopes $h_{p q}(t)$ and $h_{\tilde{p} \tilde{q}}(t)$ is defined as

$$
\rho_{p q, \tilde{p} \tilde{q}}(\tau)=\frac{\mathbf{E}\left[h_{p q}(t) h_{\tilde{p} \tilde{q}}^{*}(t-\tau)\right]}{\sqrt{\mathbf{E}\left[\left|h_{p q}(t)\right|^{2}\right] \mathbf{E}\left[\left|h_{\tilde{p} \tilde{q}}(t)\right|^{2}\right]}}
$$

where $(\cdot)^{*}$ denotes the complex conjugate operation, $\mathbf{E}[\cdot]$ is the statistical expectation operator, and $p, \tilde{p} \in\left\{1, \ldots, L_{R}\right\}$. From (1) and (7), the ST CF can be written as

$$
\begin{aligned}
\rho_{p q, \tilde{p} \tilde{q}}(\tau)= & \lim _{M, N \rightarrow \infty} \frac{1}{M N} \sum_{m=1}^{M} \sum_{n=1}^{N} E\left[e^{-j \frac{2 \pi}{\lambda}\left(\varepsilon_{p m}+\varepsilon_{q n}-\varepsilon_{\tilde{p} m}-\varepsilon_{\tilde{q} n}\right)}\right. \\
& \times e^{j 2 \pi \tau f_{T \max } \cos \left(\alpha_{T}^{(m)}-\gamma_{T}\right) \cos \beta_{T}^{(m)}} \\
& \left.\times e^{j 2 \pi \tau f_{R \max } \cos \left(\alpha_{R}^{(n)}-\gamma_{R}\right) \cos \beta_{R}^{(n)}}\right]
\end{aligned}
$$

Since the number of local scatterers in the reference model is infinite, the discrete azimuth AoDs (AAoDs) $\alpha_{T}^{(m)}$, elevation AoDs (EAoDs) $\beta_{T}^{(m)}$ and azimuth AoAs (AAoAs) $\alpha_{T}^{(n)}$, elevation AoAs (EAoAs) $\beta_{R}^{(n)}$ can be replaced with continuous random variables $\alpha_{T}, \beta_{T}$ and $\alpha_{R}, \beta_{R}$ with joint probability density functions $f\left(\alpha_{T}, \beta_{T}\right)$ and $f\left(\alpha_{R}, \beta_{R}\right)$, respectively. Hence, (8) can be rewritten as

$$
\begin{aligned}
\rho_{p q, \tilde{p} \tilde{q}}(\tau)= & \int_{-\pi}^{\pi} \int_{-\pi}^{\pi} \int_{-\pi}^{\pi} \int_{-\pi}^{\pi}\left[e^{-j \frac{2 \pi}{\lambda} A}\right. \\
& \times e^{j 2 \pi \tau f_{T \max } \cos \left(\alpha_{T}-\gamma_{T}\right) \cos \beta_{T}} \\
& \times e^{j 2 \pi \tau f_{R \max } \cos \left(\alpha_{R}-\gamma_{R}\right) \cos \beta_{R}} \\
& \left.\times f\left(\alpha_{T}, \beta_{T}\right) f\left(\alpha_{R}, \beta_{R}\right)\right] d \alpha_{T} d \beta_{T} d \alpha_{R} d \beta_{R}
\end{aligned}
$$

where $A \approx 2 d_{T}\left[\cos \beta_{T} \cos \phi_{T} \cos \left(\theta_{T}-\alpha_{T}\right)+\sin \phi_{T} \sin \beta_{T}\right]+$ $2 d_{R}\left[\cos \beta_{R} \cos \phi_{R} \cos \left(\theta_{R}-\alpha_{R}\right)+\sin \phi_{R} \sin \beta_{R}\right]$.

Different from available 3D RS-GBSMs proposed in [9] and [10], where the unrealistic assumption $f\left(\alpha_{T / R}, \beta_{T / R}\right)=$ $f\left(\alpha_{T / R}\right) f\left(\beta_{T / R}\right)$ (i.e., the azimuth angle and elevation angle are independent) is used, the proposed model jointly considers the azimuth angle and elevation angle. To this end, VMF distribution is used to characterize the scatterer distribution in this paper.

\section{B. VMF Distribution}

The VMF distribution as shown in Fig. 2 is a 3D directional distribution that jointly considers both the azimuth and elevation angles. It is defined as [12]

$$
\begin{aligned}
f(\alpha, \beta)= & \frac{k}{4 \pi \sinh k} \exp \left\{k \left[\sin \beta_{0} \cos \beta \cos \left(\alpha-\alpha_{0}\right)\right.\right. \\
& \left.\left.+\cos \beta_{0} \sin \beta\right]\right\} \cos \beta
\end{aligned}
$$


where $k(k \geq 0)$ is known as the concentration parameter that controls the concentration of the distribution relative to the mean direction identified by $\alpha_{0}$ and $\beta_{0}$. The larger the value of $k$, the more the distribution is concentrated towards the mean direction. For $k \rightarrow \infty$ the scattering becomes extremely non-isotropic, while for $k=0$ isotropic scattering occurs.

Applying the VMF distribution to the proposed 3D twosphere model, we have the following joint scatterer distributions $f\left(\alpha_{T}, \beta_{T}\right)$ for the AAoD $\alpha_{T}$ and EAoD $\beta_{T}$, and $f\left(\alpha_{R}, \beta_{R}\right)$ for the AAoA $\alpha_{R}$ and EAoA $\beta_{R}$

$$
\begin{aligned}
f\left(\alpha_{T}, \beta_{T}\right)= & \frac{k_{T}}{4 \pi \sinh k_{T}} \exp \left\{k _ { T } \left[\sin \beta_{T 0} \cos \beta_{T}\right.\right. \\
& \left.\left.\times \cos \left(\alpha_{T}-\alpha_{T 0}\right)+\cos \beta_{T 0} \sin \beta_{T}\right]\right\} \cos \beta_{T} \\
f\left(\alpha_{R}, \beta_{R}\right)= & \frac{k_{R}}{4 \pi \sinh k_{R}} \exp \left\{k _ { R } \left[\sin \beta_{R 0} \cos \beta_{R}\right.\right. \\
& \left.\left.\times \cos \left(\alpha_{R}-\alpha_{R 0}\right)+\cos \beta_{R 0} \sin \beta_{R}\right]\right\} \cos \beta_{R} .
\end{aligned}
$$

In fact, it is difficult to derive the closed-from expressions for (9). Therefore, the numerical integration method will be used to evaluate the ST CF in the next section.

\section{Numerical Results And Analysis}

In this section, based on the derived ST CF in Section III, we will study the impact of the environment parameters and elevation angles on the ST correlation properties.

\section{A. Impact of Environment Parameters}

In the developed 3D two-sphere model, the parameters $k_{T}$, $k_{R}, \alpha_{T 0}, \beta_{T 0}, \alpha_{R 0}$, and $\beta_{R 0}$, are related to the distribution of effective scatterers, so they are called environment parameters. Since the concentration parameters $k_{T}$ and $k_{R}$ determine the concentration of the effective scatterers, the larger the value of $k_{T}$, the more the concentration of the effective scatterers towards the mean $\mathrm{AAoD} \alpha_{T 0}$ and $\mathrm{EAoD} \beta_{T 0}$, and the larger the value of $k_{R}$, the more the concentration of the effective scatterers towards the mean AAoA $\alpha_{R 0}$ and EAoA $\beta_{R 0}$. Therefore, we will investigate the impact of these parameters on ST CF by adjusting the values of these parameters.

Figs. 3-5 clearly show the impact of environment parameters and antenna spacing on ST correlation properties. The main parameters used in Figs. 3-5 are listed as follows: $\lambda=0.06 \mathrm{~m}, f_{\text {Tmax }}=f_{R \max }=500 \mathrm{~Hz}, \theta_{T}=\theta_{R}=\frac{\pi}{4}$, and $\gamma_{T}=\gamma_{R}=0$.

Both Figs. 3 and 4 adopt the antenna spacings $d_{T}=d_{R}=$ $\lambda$. For the rest parameters, Figs. 3 and 4 are obtained with $\alpha_{T 0}=\beta_{T 0}=\alpha_{R 0}=\beta_{R 0}=0$ and $k_{T}=k_{R}=10$, respectively. Therefore, Fig. 3 shows the ST correlation properties with different values of $k_{T}$ and $k_{R}$. Fig. 4 illustrates the ST correlation properties with different mean AoDs $\alpha_{T 0}$ and $\beta_{T 0}$, and different mean AoAs $\alpha_{R 0}$ and $\beta_{R 0}$.

From Figs. 3 and 4, it is clear that these environment parameters significantly affect ST correlation properties. Fig. 3 tells us that the increase of the value $k_{T}$ and $k_{R}$ will enhance the ST correlation. Moreover, if $k_{T}$ and $k_{R}$ use the same set of values (e.g. $k_{T}=0, k_{R}=20$ or $k_{T}=20, k_{R}=0$ ) the ST correlations are identical. In Fig. 4, we note that the ST correlations are equivalent with $\beta_{T 0}=\beta_{R 0}=0$, no mater the values of $\alpha_{T 0}$ and $\alpha_{R 0}$. In this case, received powers mainly come from horizontal plane. Whereas, the non-zero mean $\operatorname{EAoD}\left(\beta_{T 0} \neq 0\right)$ and $\operatorname{EAoA}\left(\beta_{R 0} \neq 0\right)$ indicate received powers mainly come from either tall buildings $\left(\beta_{T 0 / R 0}>0\right)$ or grounds $\left(\beta_{T 0 / R 0}<0\right)$. Overall, based on the developed 3D M2M channel model and VMF distribution, $\alpha_{T 0}, \beta_{T 0}, \alpha_{R 0}$ and $\beta_{R 0}$ significantly affect the ST correlation properties.

Fig. 5 shows the ST correlation properties for different values of antenna spacing and the rest parameters are $k_{T}=$ $k_{R}=10$ and $\alpha_{T 0}=\beta_{T 0}=\alpha_{R 0}=\beta_{R 0}=0$. From Fig. 5, we observe that the increase of the antenna spacings $d_{T}$ and $d_{R}$ will decrease the ST correlation. This agrees with our intuition that the larger the antenna spacing, the smaller the spatial correlation between the antenna elements, and thereby the higher the capacity. For the special case $d_{T}=d_{R}=0$, a SISO scenario occurs in reality, which has the highest correlation. Note that in this case, the ST CF reduces to a time CF only.

\section{B. Impact of Elevation Angles}

In what follows, we will analyze the impact of the elevation angles $\beta_{T}$ and $\beta_{R}$ on the $\mathrm{ST}$ correlation properties by adjusting the ranges of these two elevation angles.

To investigate the impact of elevation angles on ST properties, we assume the antenna spacing $d_{T}$ equals to zero with the different ranges of angles $\beta_{T}$ and $\beta_{R}$. The parameters used in Figs. 6 and 7 are listed as follows: $\lambda=0.06 \mathrm{~m}$, $f_{\text {Tmax }}=f_{R \max }=500 \mathrm{~Hz}, k_{T}=k_{R}=50, d_{R}=10 \lambda$, $\theta_{T}=\theta_{R}=\frac{\pi}{4}, \phi_{T}=\phi_{R}=\frac{\pi}{4}, \gamma_{T}=\gamma_{R}=0$, $\alpha_{T 0}=\alpha_{R 0}=0$, and $\beta_{T 0}=\beta_{R 0}=0$.

Figs. 6 and 7 show the ST correlation properties for different ranges of angles $\beta_{T}$ and $\beta_{R}$. Observations from Figs. 6 and 7 tell us that the increase of the range of elevation angle reduces the ST correlation properties. In other words, the smaller ranges of elevation angles, the higher correlation properties. Therefore, we can conclude that the impact of elevation angles should not be neglected for accurately capturing the ST correlation properties of real M2M channels.

In order to clearly show the impact of elevation angles on the 3D ST correlation properties, we draw them in the corresponding 2D figure as shown in Fig. 8. Meanwhile, we still change the ranges of elevation angles. The parameters used in Fig. 8 are the same as those used in Figs. 6 and 7. For comparison purposes, the zero elevation angles are also plotted in Fig. 8. In this case, it means that the twosphere model reduces to a two-ring model. From Fig. 8, we can observe that the elevation angles significantly affect the ST correlation properties. Fig. 8 also clearly tells us that the decrease of the ranges of elevation angles increases the ST correlation. This interesting observation means that the design of MIMO M2M systems based on a 2D channel model will show over-pessimistic performance as the vertical diversity, which can be further used to improve the performance of M2M systems, cannot be captured by a 2D M2M channel model. Again, this allows us to conclude that 3D MIMO M2M 
channel models are necessary for the accurate modeling of correlation properties of real M2M channels.

\section{CONCLUSION}

This paper has proposed a novel 3D two-sphere MIMO M2M RS-GBSM (only double-bounced rays), from which the ST CF has been derived. We have also investigated the impact of the environment parameters and the elevation angles on the ST correlations of non-isotropic scattering MIMO M2M channels. From the numerical results, it has been shown that the correlation properties significantly vary with the changes of the important environment parameters or the elevation angles. From these interesting observations and comparing with the corresponding 2D MIMO M2M channel model, we can conclude that 2D models show higher ST correlations than real channels. Consequently, the novel 3D MIMO M2M channel model is more accurate to reflect a real communication environment and necessary for the better design of future MIMO M2M communication systems.

\section{ACKNOWLEDGMENT}

The authors acknowledge the support from the RCUK for the UK-China Science Bridges Project: R\&D on (B)4G Wireless Mobile Communications. Y. Yuan, C.-X. Wang, and D. I. Laurenson acknowledge the support from the Scottish Funding Council for the Joint Research Institute in Signal and Image Processing, as part of the Edinburgh Research Partnership in Engineering and Mathematics (ERPem). X. Cheng acknowledges the support from Important National Science \& Technology Specific Project of China (grant no.: 2009ZX03002-012). X. Ge acknowledges the support from the National Natural Science Foundation of China (NSFC) (grant no.: 60872007), National 863 High Technology Program of China (grant no.: 2009AA01Z239), and Ministry of Science and Technology (MOST) of China for the International Science and Technology Collaboration Program (grant no.: 0903). F. Zhao acknowledges the support from the NSFC (grant no.: 60872022). C.-X. Wang and F. Zhao acknowledge the support of the Key Laboratory of Cognitive Radio and Information Processing (Guilin University of Electronic Technology), Ministry of Education.

\section{REFERENCES}

[1] C.-X. Wang, X. Hong, X. Ge, X. Cheng, G. Zhang, and J. S. Thompson, "Cooperative MIMO channel models: a survey," IEEE Commun. Mag., vol. 48, no. 2, pp. 80-87, Feb. 2010.

[2] C.-X. Wang, X. Hong, H. Wu, and W. Xu, "Spatial temporal correlation properties of the 3GPP spatial channel model and the Kronecker MIMO channel model," EURASIP Journal on Wireless Communications and Networking, vol. 2007, Article ID 39871, 9 pages, 2007. doi:10.1155/2007/39871.

[3] C.-X. Wang, X. Cheng, and D. I. Laurenson, "Vehicle-to-vehicle channel modeling and measurements: recent advances and future challenges," IEEE Commun. Mag., vol. 47, no. 11, pp. 96-103, Nov. 2009.

[4] A. S. Akki and F. Haber, "A statistical model for mobile-to-mobile land communication channel," IEEE Trans. on Veh. Technol., vol. 35, no. 1, pp. 2-10, Feb. 1986.

[5] A. S. Akki, "Statistical properties of mobile-to-mobile land communication channels," IEEE Trans. on Veh. Technol., vol. 43, no. 4, pp. 826-831, Nov. 1994

[6] M. Pätzold, B. O. Hogstad, and N. Youssef, "Modeling, analysis, and simulation of MIMO mobile-to-mobile fading channels," IEEE Trans. Wireless Commun., vol. 7, no. 2, pp. 510-520, Feb. 2008.

[7] A. G. Zajić and G. L. Stüber, "Space-time correlated mobile-to-mobile channels: modeling and simulation," IEEE Trans. on Veh. Technol., vol. 57, no, 2, pp. 715-726, Mar. 2008.
[8] X. Cheng, C.-X. Wang, D. I. Laurenson, S. Salous, and A. V. Vasilakos, "An adaptive geometry-based stochastic model for non-isotropic MIMO mobile-to-mobile channels," IEEE Trans. on Wireless Commun., vol. 8, no. 9, pp. 4824-4835, Sep. 2009.

[9] A. G. Zajić and G. L. Stüber, "Three-dimensional modeling, simulation, and capacity analysis of space-time correlated mobile-to-mobile channels," IEEE Trans. on Veh. Technol., vol. 57, no. 4, pp. 2042-2054, Jul. 2008.

[10] A. G. Zajić and G. L. Stüber, "Three-dimensional modeling and simulation of wideband MIMO mobile-to-mobile channels," IEEE Trans. on Wireless Commun., vol. 58, no. 3, Mar. 2009.

[11] X. Cheng, C.-X. Wang, Y. Yuan, D. I. Laurenson and X. Ge, "A novel 3D regular-shaped geometry-based stochastic model for non-isotropic MIMO mobile-to-mobile channels," invited paper, Proc. IEEE VTC '10-Fall, Ottawa, Canada, Sep. 2010, accepted for publication.

[12] K. V. Mardia and P. E. Jupp, Directional Statistics, Chichester: John Wiley\&Sons, 2000.

Table 1. Definition of parameters in Fig. 1.

\begin{tabular}{|c|l|}
\hline$D$ & distance between the centers of the Tx and Rx spheres. \\
\hline$R_{T}, R_{R}$ & radius of the Tx and Rx spheres, respectively. \\
\hline$d_{T}$ & spacing between the $p^{t h}$ and $\tilde{p}^{t h}$ antenna elements at the Tx. \\
\hline$d_{R}$ & spacing between the $q^{t h}$ and $\tilde{q}^{t h}$ antenna elements at the Rx. \\
\hline$\theta_{T}, \theta_{R}$ & $\begin{array}{l}\text { azimuth angles of the } p^{t h} \text { transmit and } q^{t h} \text { receive } \\
\text { antenna element (relative to the } x-y \text { plane), respectively. }\end{array}$ \\
\hline$\phi_{T}, \phi_{R}$ & $\begin{array}{l}\text { elevation angles of the } p^{t h} \text { transmit and } q^{t h} \text { receive } \\
\text { antenna element (relative to the } x-y \text { plane), respectively. }\end{array}$ \\
\hline$\alpha_{T}, \alpha_{R}$ & $\begin{array}{l}\text { azimuth angles of departure (AAoD) and the azimuth } \\
\text { angles of arrival (AAoA), respectively. }\end{array}$ \\
\hline$\beta_{T}, \beta_{R}$ & $\begin{array}{l}\text { elevation angles of departure (EAoD) and the elevation } \\
\text { angles of arrival (EAoA), respectively. }\end{array}$ \\
\hline$v_{T}, v_{R}$ & velocities of the Tx and Rx, respectively. \\
\hline$\gamma_{T}, \gamma_{R}$ & moving directions of the Tx and Rx, respectively. \\
\hline$\varepsilon_{p m}, \varepsilon_{\tilde{p} m}, \varepsilon_{m n}$, & $\begin{array}{l}\text { distances } d\left(A_{T}^{(p)}, S_{T}^{(m)}\right), d\left(A_{T}^{(\tilde{p})}, S_{T}^{(m)}\right), d\left(S_{T}^{(m)}, S_{R}^{(n)}\right), \\
d\left(A_{R}^{(q)}, S_{R}^{(n)}\right) \text { and } d\left(S_{R}^{(n)}, A_{R}^{(\tilde{q})}\right), \text { respectively. }\end{array}$ \\
\hline$\varepsilon_{q n}$ and $\varepsilon_{\tilde{q} n}$ & distances $d\left(S_{T}^{(m)}, C\right)$ and $d\left(S_{R}^{(n)}, C^{\prime}\right)$, respectively. \\
\hline$h_{T}$ and $h_{R}$ &
\end{tabular}

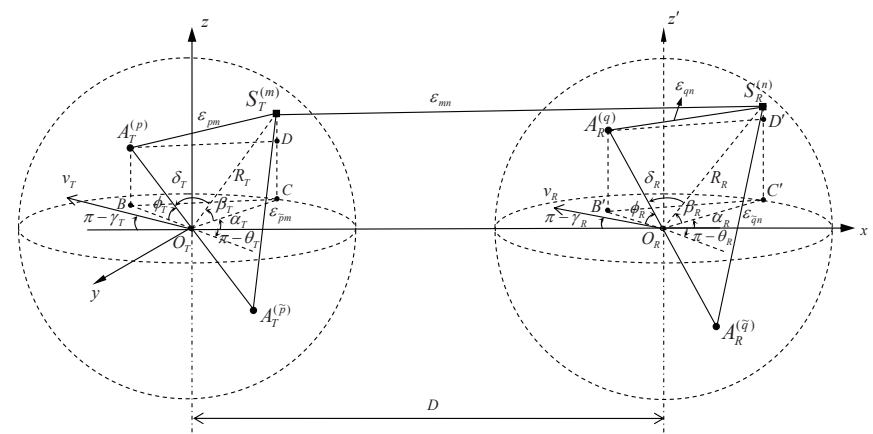

Fig. 1. The 3D two-sphere model for MIMO M2M channels.

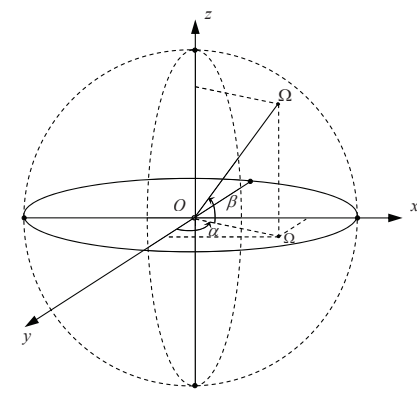

Fig. 2. VMF distribution in a 3D system. 


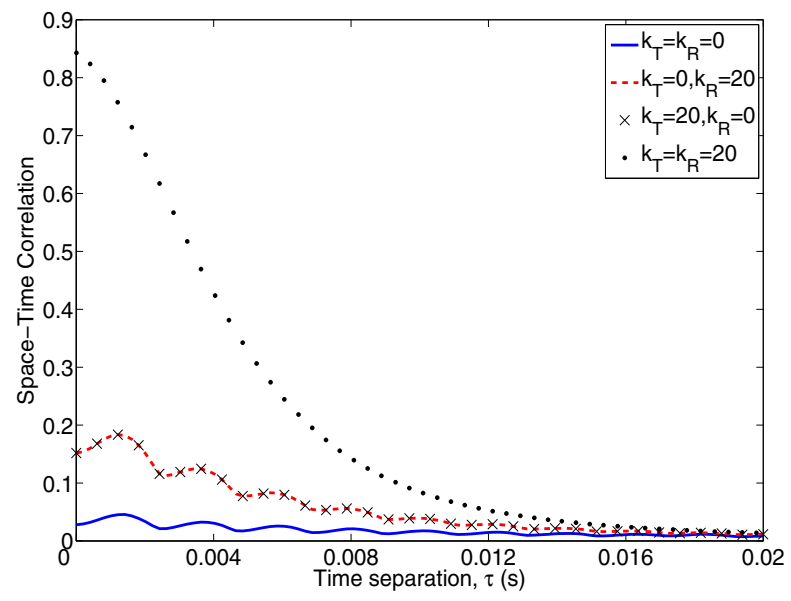

Fig. 3. The ST CF with different $k_{T}$ and $k_{R}$.

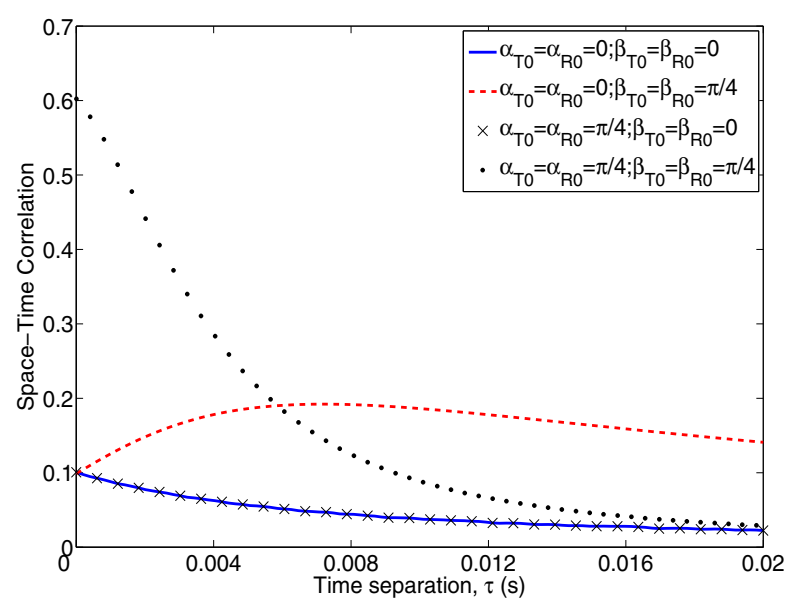

Fig. 4. The ST CF with different $\alpha_{T 0}, \beta_{T 0}, \alpha_{R 0}$, and $\beta_{R 0}$.

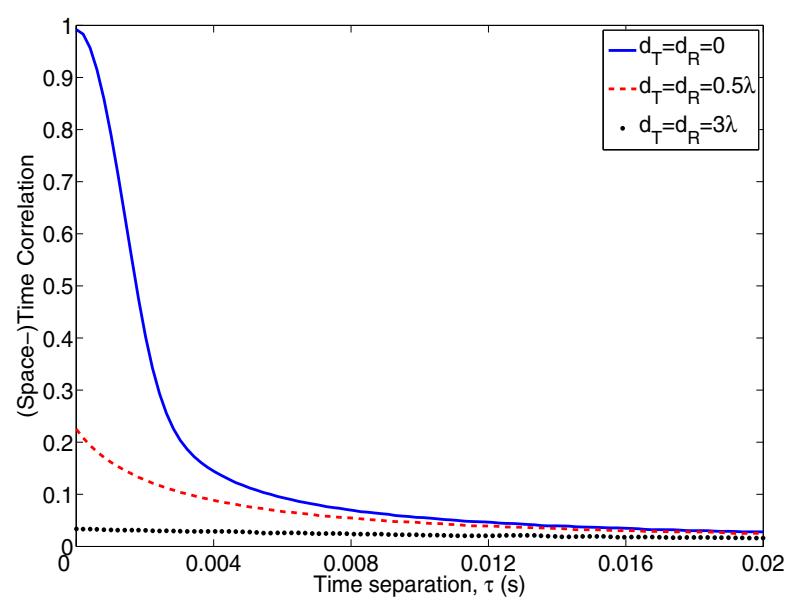

Fig. 5. The ST CF with different $d_{T}$ and $d_{R}$.

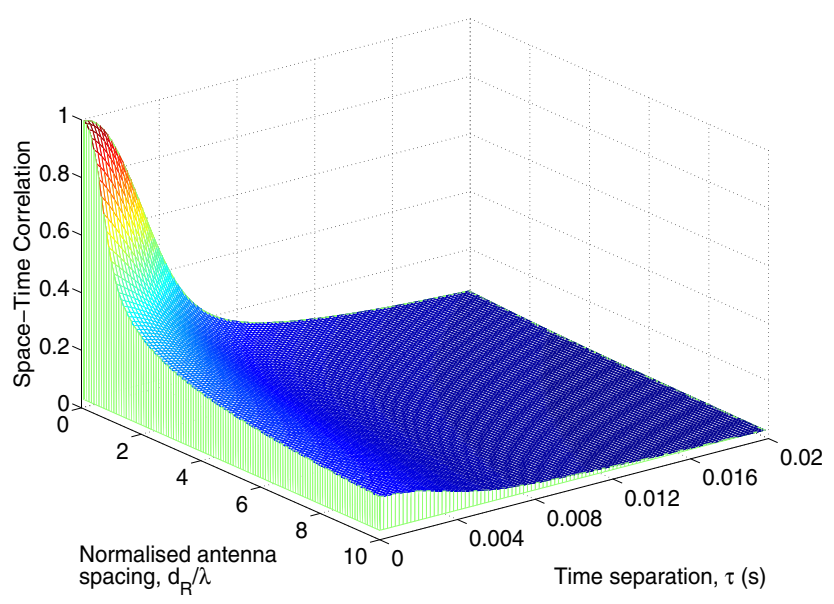

Fig. 6. The 3D ST CF $\left(\beta_{T}\right.$ and $\left.\beta_{R} \in[-\pi / 8, \pi / 8)\right)$.

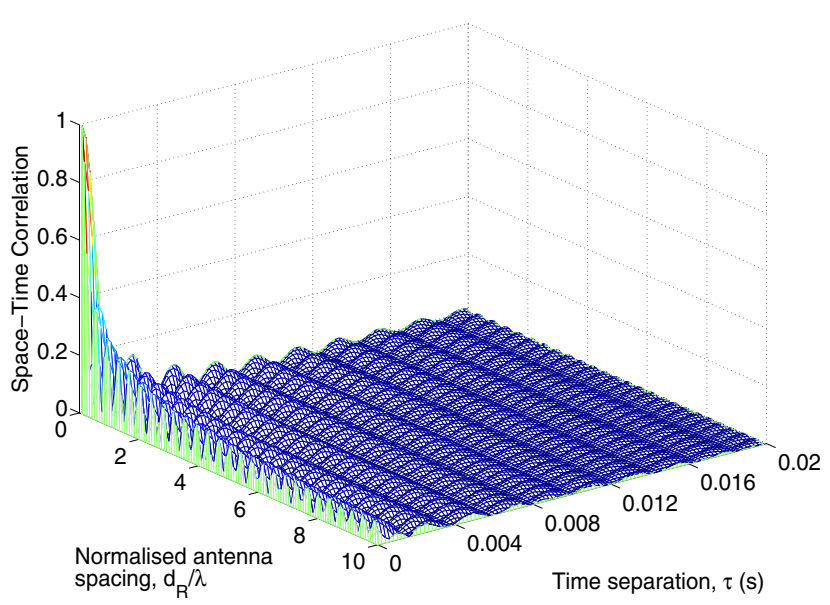

Fig. 7. The 3D ST CF $\left(\beta_{T}\right.$ and $\left.\beta_{R} \in[-\pi, \pi)\right)$.

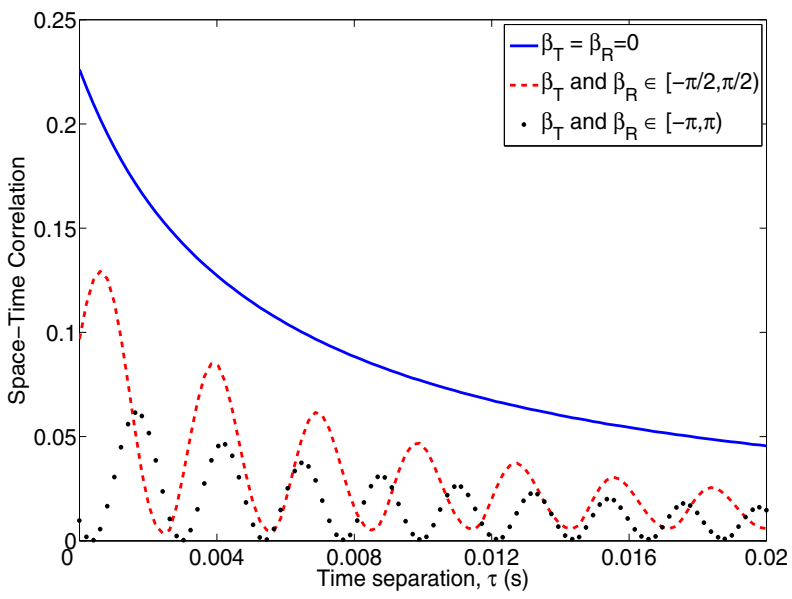

Fig. 8. The ST CF with different ranges of $\beta_{T}$ and $\beta_{R}$. 